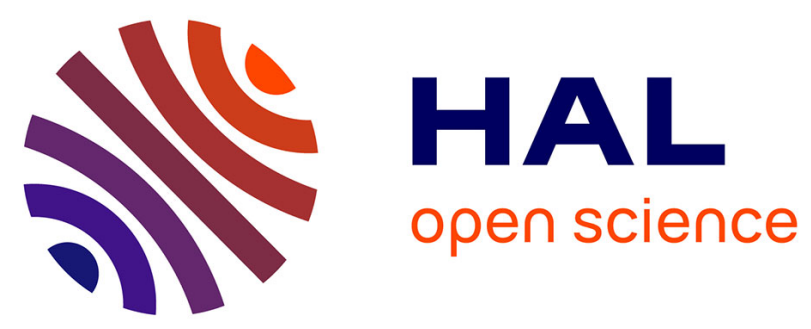

\title{
La mosaïque de Tourmont (Jura)
}

Henri Stern

\section{To cite this version:}

Henri Stern. La mosaïque de Tourmont (Jura). Gallia - Fouilles et monuments archéologiques en France métropolitaine, 1961, 19 (2), pp.248-254. 10.3406/galia.1961.2328 . hal-01926099

\section{HAL Id: hal-01926099 \\ https://hal.science/hal-01926099}

Submitted on 25 Feb 2020

HAL is a multi-disciplinary open access archive for the deposit and dissemination of scientific research documents, whether they are published or not. The documents may come from teaching and research institutions in France or abroad, or from public or private research centers.
L'archive ouverte pluridisciplinaire $\mathbf{H A L}$, est destinée au dépôt et à la diffusion de documents scientifiques de niveau recherche, publiés ou non, émanant des établissements d'enseignement et de recherche français ou étrangers, des laboratoires publics ou privés.

\section{(이) $\$$}

Distributed under a Creative Commons Attribution - NonCommercial - NoDerivatives $\mid 4.0$ 
les aul res libules indiequenl une dale voisine du debul de l'bimpire (er-ate siecles de nolre ire) pour ce rimelieres qui liva auparavant des objels plus anciens de l'époque de la lione, nol amment des armilles filiformes el des fibules du lype en tenailles. ainsi que des pendeloques-crolales (fig. 5). Cependant. pour ne pas se meprendre sur la question relromologique. il comviont de rappeler qu'un certain comserva- lisme cullurel se manifeste a loules les cipoques dans res regions alpestres.

I rote des fibules. les objels romprennent des rouleaux en fer. des clous en fer. diverses chatonetles en bronze. quelques appliques in lóle de bronze, el des perles en pale de verre bleu, du lype courant dans loules les régions.

\section{J.-Cil. Colments.}

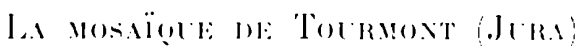

On a découvert. il y a environ 20) ans, en 1711 ou 1717 pres d'un village du Jura. Toumont arrondissement de Poligny au Cord de Lons-le-saunier au lieu-dil Les C.hambrelles on Eslabaye. a liol milres environ alu SordOuest du village. sur le bord de la roule de bole. une mosaïgue romaine qui allia l'allen-

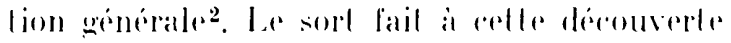
a de un pen plus favorable que dans bien d'alleres ras du memes gente. Ce pavement. enfoui une premiere fois par l'inventeur. le lermier Fleur de Tourmonl. pour le proleger contre les delépioralions par des visileurs indiserels. ful degage a nouveau à l'oceasion d'une visile de l'historien I)unod de Ciharnage. de Besancon, yui vinl le voir en ortobre 17.6103. Reste probablement a ciel ouvert justu'en seplembre 17\%), il ful alors lobjel d'une autre visite de personnages d'une certaine importance, de l'historien de Poligny. Fr.-F. Chevalier, ef du Marquis de Montrichardt. Gràce à ces deux visiles.

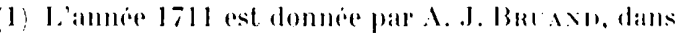
som rapport de la decouverle, paru dans l'Almuaire de

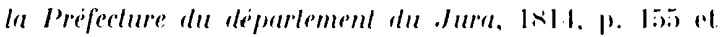

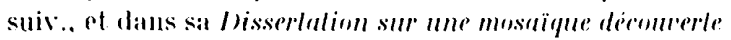
pres de la rille de Poligny. Patris, lisli, p. i, alors que

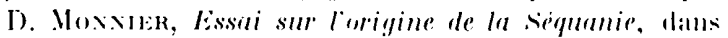
Almanarh historique el agronmmique de la l'refeclure du . Jura pour lamnee 1819, p. 191 et suiv., indique 1717.

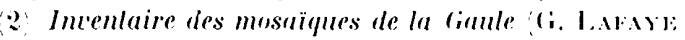

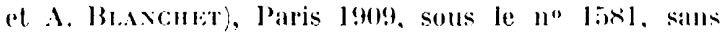
illustration. Portera le no 316 dans. H. Srters, Recueil gencral des musaïques de la Ciaule, t. I. 3. a jaraitre.

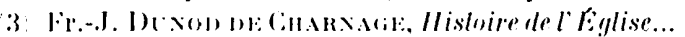
ar Besancon. I I. 17501, 1. 35: a a 359.

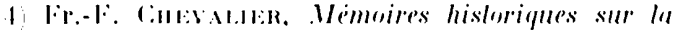

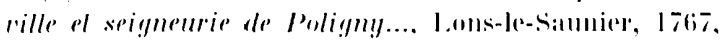
p. I.X.X i $1: 11$. nous an possedons deux descriptions assez delaillées, el qui plus est. une pravure que Caylus a publice au lome IV de son Recueil d'anliquiles. d'apres un dessin transmis par le marequis de Montrichard the. 1 Par la suite el loujours dans le meme but de protection contre l'ondommagement par les visilems, la mosaïyue lut entouie de nomrau. I en reroire 1). Bomniere on l'aurail dégagée encore une fois en list: peut-ible dans l'intention de l'enlever el de la placer dans le musée fonde alors a lons-le-samnier. Nais on en resta aux projets. Ce documenl remarquable de larl romain en Gaule ne sortil pas de lerre of y est ancore de nos jours. A la fin du siecle passe un homonyme. de 1). Momnier. Pierre Momnier, fit ampagne. pour une fouille mélhodique. sans résullal?

Vous iqnorons dans quel étal se troure aujourd'hui celle mosaïque, qui, a en croire les témoins orulaires, élail parfailement conservere all moment de sa découverte. Les mulliples operalions de degagement el d'enfouissement, les prelievements de cubes par les visileurs. noul cerles pas manqué de la delériorer, mais il semblemail qu'une partie imporlanle en existe encore. It serait possible de la mellme all jour.

le prime abord l'elude d'un parement qui ne nous est eonmu que par des descriptions et par une gravure du xime siocle parait offrir

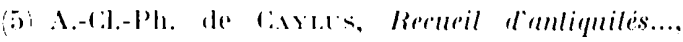
IV. Paris, 1761, pl. t:Xill.

(i) lone. ril.

7 l. Moxwlis. la mostüque gallo-romaine de Tourmunl pres de Polign!y (.lura). daus Soles darl

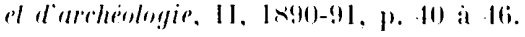




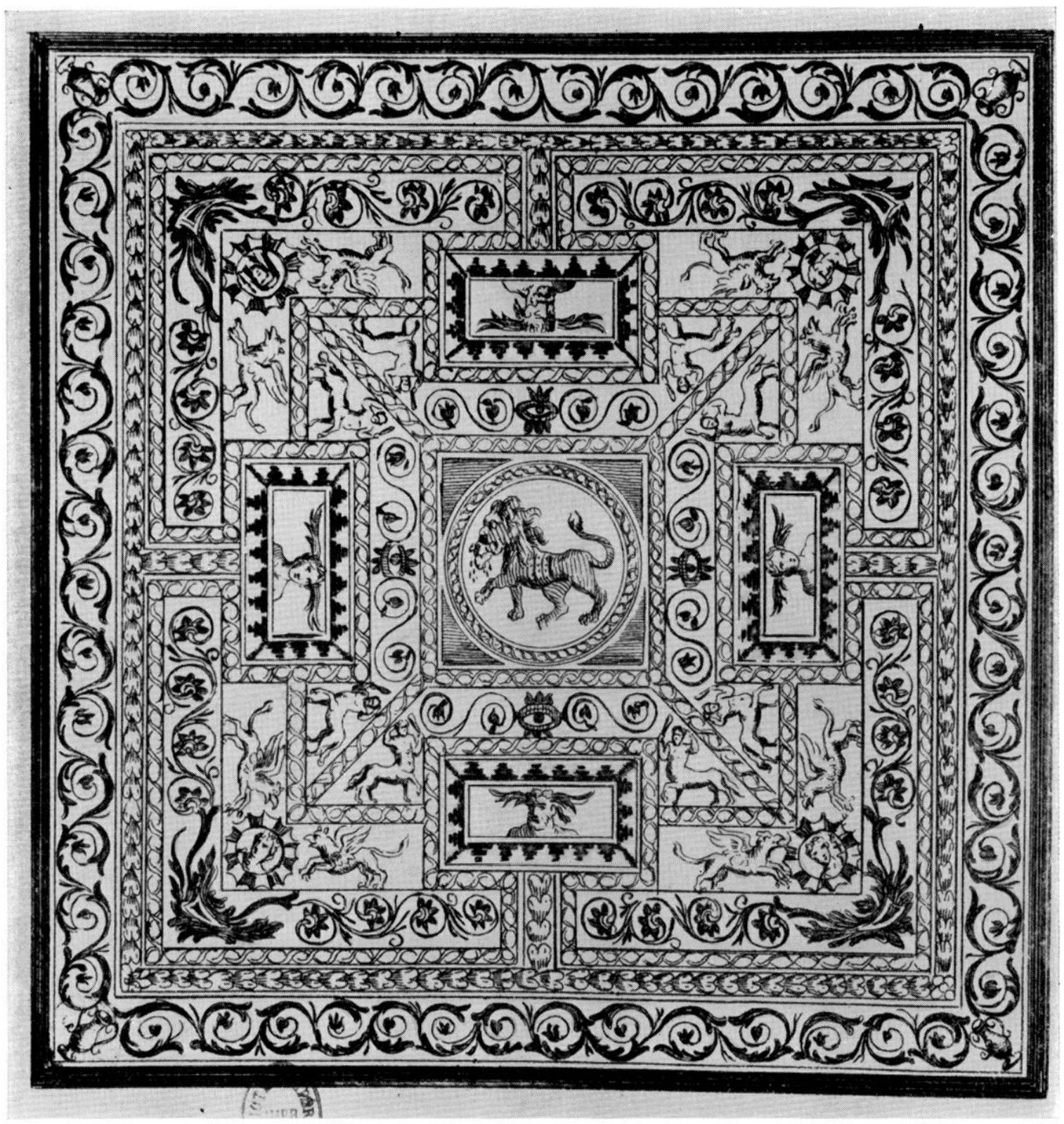

Fig. 1. -... Wosaique de Tourmont Cartrs, Recueil danliquiles. IV. pl. CXXIII.

un intérì réduil. Ces témoignages anciens. apparemment consciencieux. permetlent rependant d'en expliquer l'iconographie. incomprise jusqu'ici. el de le dater dans des limiles chronologiques assez étroiles. chose l ress rare pour les mosaïques gallo-romaines.

Los renseignements sur le plan de la villa. dont celle mosaïque ornail une piece de 20) pieds (6 m. 50) au rarré. sonl vagues. On avail degagé les fondations d'un long portique et d'une vaste piece dentrée. lous deux parés de plaques de marbre blane, suivis de six pieces plus petites réparties par groupes de trois de part el d'autre (a l'list el a l'ouest d'un couloir. Les trois pieces de l'Ouest élaient. pavées de plaques de marbre de couleurs variées, celles de l'Est, de mosaïques. Le pavement d'une seule de res derniores élail ronserve: rest celui qui nous occupe.

In coup d'ril sur la planche de Caylus (fig. 1 sullit a montrer que la composition de celle mosaïque est differente de toules celles que 


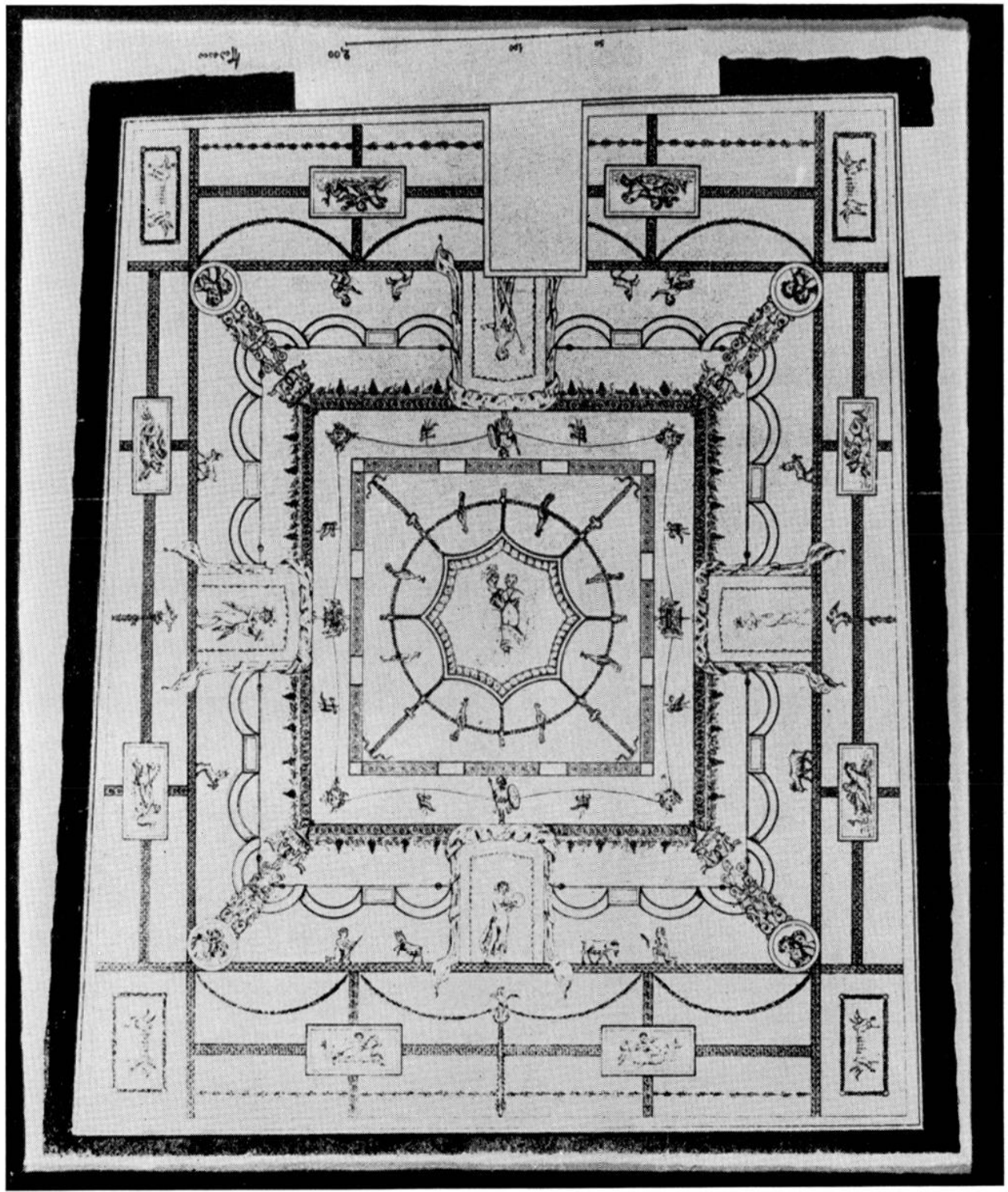

Fir. : . Pompiö. Plafond peint.

l'on comnait dans la provine de Belgique. Bille imite le décor d'un plafond. looro lexis el. plus récemment. (i.-ch. Picard ${ }^{9}$ onl réuni un eroupe de mosal̈ques de pavement. peru nombreuses, distanles dans l'espace el dans le lemps. qui reproduisent les decors des plafonds. la notre est particulierement proche d'un plafond peint de Pompei qui appartient au

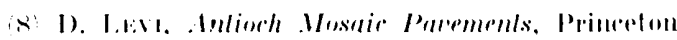

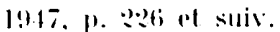

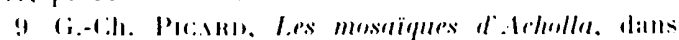

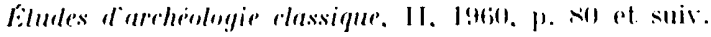

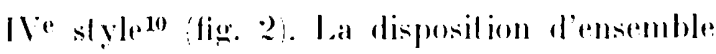
rst a peu pris la mime : au centre un médaillon rirculaire qui. rhargé a Pomperi d'un voile fendu. contient une ligure. Il est entouré de rarres inscrits les uns dans les aul res el relies par des bandes diagonales. I e milieu de chaque roble de l'un des rarres est rharge d'un lableaulin qui renferme une represenlation figurée. Ies angles exterienurs, d'ou partent les dia-

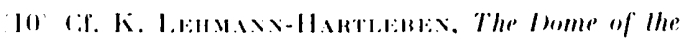

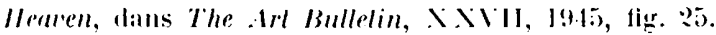


Enonales. soml ranlommes de medaillons reilrulaires qui renterment des lales. Vul deulde

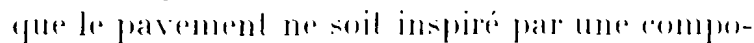
silion de ce gente. Ia densile du derore a Tourmont. qui ne laisse apparaitre que des parties restreintes du fond. interdil rependant de penser pour la mosä̈que a une dérivation direcle de ces plafonds qui sont plus anciens diun sienle el demi environ.

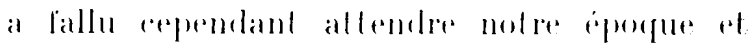

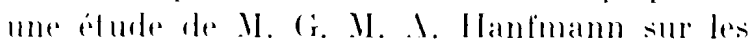
representalions anligues des saisons pour comprendre la significalion des qualle feles dans les radres reetangulaires. pourveres d'ailes verdatres allachées aux lempes. Ce ne sont pas des dieux du pantheon enero-romain. ere que pensaient les auleurs du xine siecle mais les persomnificalions des rents. Le lait est civident.

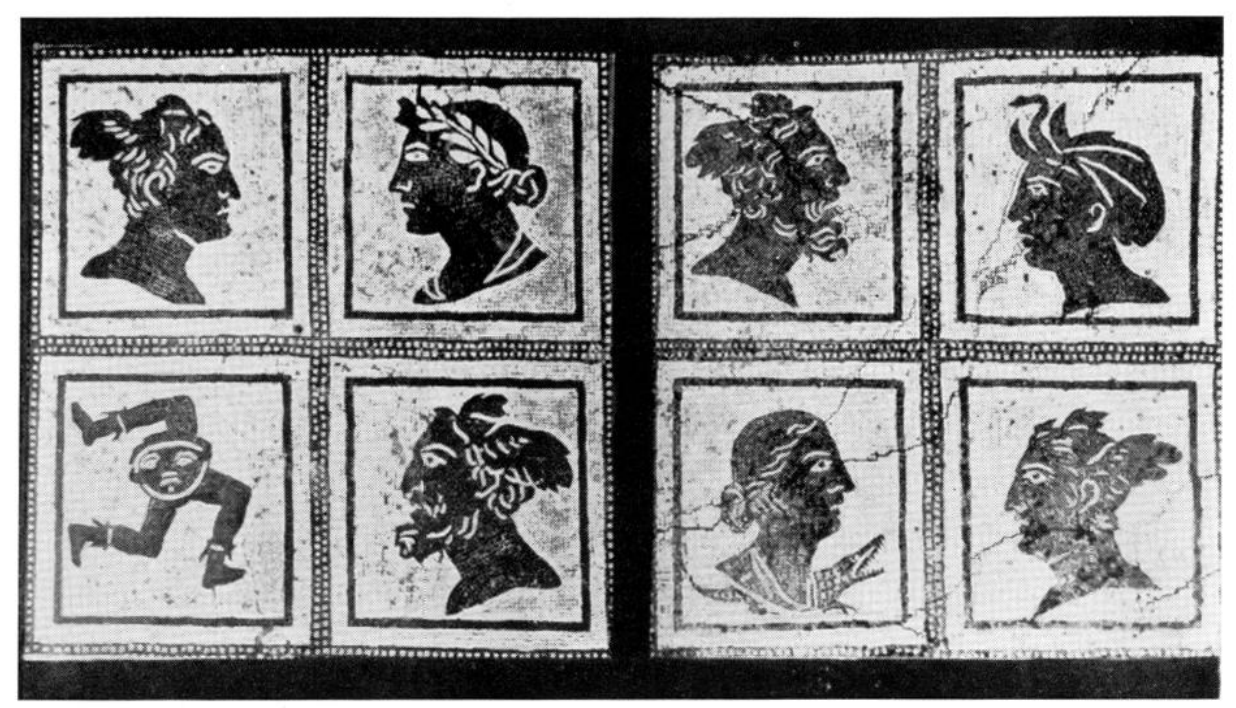

Fir. 3. - Bustes des Vents et des Provinces. Mosaique dostie MLкк. I, pl. 49.

Les sujels représenles avaient suscilé la plus grande curiosile au moment de la découverle. On s'élait laissé aller a des spéculations curieuses ef fantaisistes. Dunod pensait y reconnaile des allusions a l'hisloire de Virwinie : le lion enchainé au centre serail Ippius Claudius, la brebis qu'il dévore. l'innocente Virginie, les centaures, des chevaliers romains. le commentaire de Chevalier. voyant une représentation symbolique de l'étal pacifié de la séquanie sous le rigne heureux d'Intonin le Pieux, n'aura pas, je le crains, plus de chance d'être agrée aujourd'hui. (Juoiqu'il en soit. l'iconographie de celle mosalyue est assez particuliere et. a certains égards. unique.

Seuls les bustes des quatre saisons dans les angles, placés dans des médaillons el affrontes chacun par deux grifons. ont toujours été reconnus comme lels. Less attributs qu'ils portent, indistincls sur la cravure. mais décrits par lounod et Chevalier, en font foi. Il ef ne demande pas de demonstration. Inepuis le ${ }_{1}$ e siecle de notre ire (une dale plus précise ne peul itre donnée, l'iconographie des vents n'ayant pas lait l'objet de l'élude quelle merile: les vents sont habituellement. representes par un groupe de quatre bustes masculins. dont deux barbus et deux imberbes (iy. 3:12. Ies leles barbues sont celles des vents froids de l'Est el du Nord. Eurus et Bareas. les deux aulres ceux de l'ouest el du Midi, Kephyrus et Nolus.

Leur association avec les saisons, sans itre frequente, se trouve sur quelques monuments

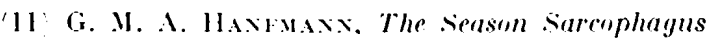
in Mumbarton Oals, Cambridgre. Massachussets, 1951, I. p. 252 et suiv.. II. p. 152, n" 169.

12 sur les representations des lents, ibid.. et F. Conost. Recherches sur le sumbulisme funeraire des Romains. Paris. 1912. p. 106. n. 5. p. 107. n. 2. p. 151 et suiv.. et pressim. 


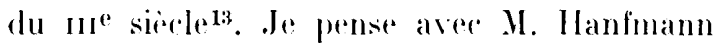
qu'elle est toule superficielle el depourvue de signifiealion profomele : les vents doux el rhauds acoompagnent te Prinlemps el l'bié. les froids. l'Aulomne al l'lliver. I al révolution saisomniere, lopos favori de l'arl romain depuis les Antonins. est le sujel principal de colle mosaïgute.

Dans re contexle. la presence du lion au rentre est surprenante : il introduil une nole inhabiluelle dans une ieonographie par ailleurs banale. II. Hanfmann, seul parmi les areheologues modernes qui ail domné un avis sur celle image, suggere. pour l'expliquer. quelque rare mythe rosmique oriental sans previser. en se référant aux études de F. Cumont sur le symbolistme funérairedt. Cel animal se distingue par deux particularilés : il lient une léte de bélier ou de brebis degoullante de sang dans la queule el son corps est enserre par "une bande de reuir"s. renforede el ormere par des

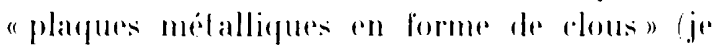
rile Dunod de Charnagere qui soest inleresse a re delail. Or. ces deux parliculariles se relrourent réunies dans certains exemplaires d'un eroupe des sareophages romains, décorés aux angles de lions oul de protomes de lion (liz. 1). Iess faures salisissenl de lemes palless avanl unc victime. sanglier. onagre. cert. bour ou belier. qu'ils sappreitent a devorer. Iener

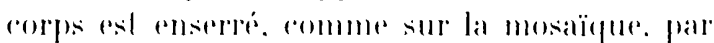
une sangle allachere i lun garrol qui enloure

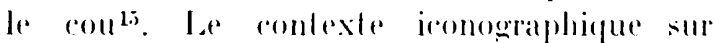

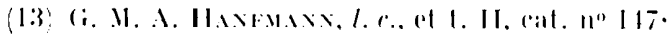

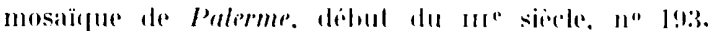
mosaïlue de kabr-lliram. fin lu ve siocle, no 311.

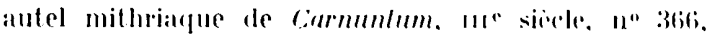

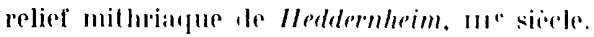

(14) O. 1., 11. 25:3.

(15) Voici les ciny sareophages sur lesplurls jai pu

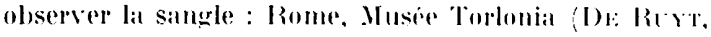
voir in/ra, note 19, lig. loi : Izome, jalrdin du Palats

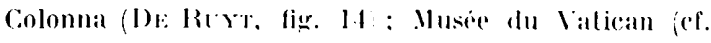

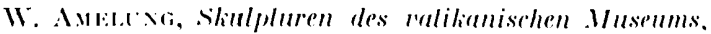

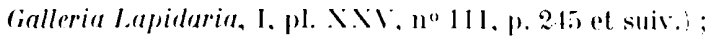

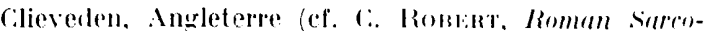
phagi al Clieveden, dalls .Journal of Hellenir sludies.

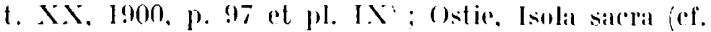

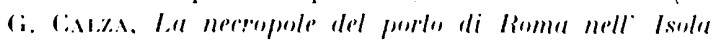

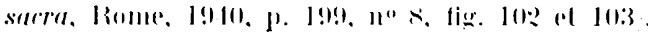

quelques-uns de ces sareophages fail aisement comprendre ce detail. Les lions sonl aceompat gnes d'un arardien ou d'un ehasserur qui les harcibe ou les incile avere un pien ou une pique (fig. f) ${ }^{16}$ : ee sonl des faures de l'arime qu'on avail l'habilude de harnacher ainsiti La ressemblance avere le lion de la mosaïgue est frappante: irei al li, un faure do ciryur arhere sa vielime.

()n a beaucoup discule sur la signification de ces lions. Fe. Cumonter el Fr. De Ruyll". ne les ronnaissant que par les sareophages leur donnaient un sens funceraire : ils symboliseraient la puisiance de la morl qui enlive l'homme sans qu'il puisse résister2). Or, on ne saurail admelle celle inlerprétalion pour nolre mosaïque qui, de loule évidence: n'est pas funéraire. (i. Rodenwaldl, dans une belle elude stur quelques sarcophages de ce groupe. en propose une autre qui me paraîl. preferable : le lion serail l'image de la foree vilale el prendrait. de ce fait. un caractiere apotropaïque. qui est ronfirmé d'alleors par

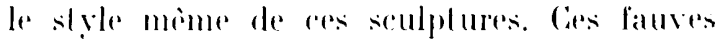
majestueux sont romme les gardiens de la lomber. deoi ils repoussent les forees nocives.

Le gont de patheligue al de l'action dramalique. arareleristique de l'art romain de l'eperque dess severess sans avoir reré re molil qui remonte i des modeles orienlate du vine al du

16, I.a plupart des lioms harnachios sont accomplagrtes d'un servant ; seuls cens du sareophage de l’Isolat sacrat font exception. Par rontere sur lut assez gratul mombre de sareophaters. des lioms nom-hammachis sont suivis de semints.

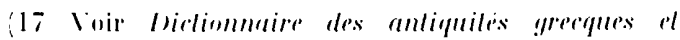
romaines. s. v. enenalin. p. 703.

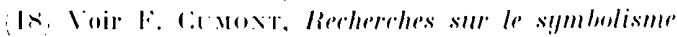
fumirate... 1. 15:

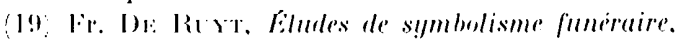
dans Bulletin de I Instilul hislorigue belge de Rome. NIIT, 1936 . p. 169 a 175.

:0: J. (1) astex, He Cimbinschrift des Besalits. Vilaloras "Libera eos de ore leomis", dans Römische Villeilungen, I.I II, 1933. p. 50 a 69, a puldie une plaque de lerulus chretienne, oin cette signifieation du lion pst assure par l'inscription. Mais il y symbolise les forees du mal dans un sens striclement chrotieri.

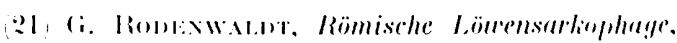

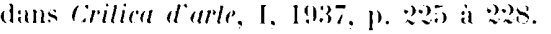




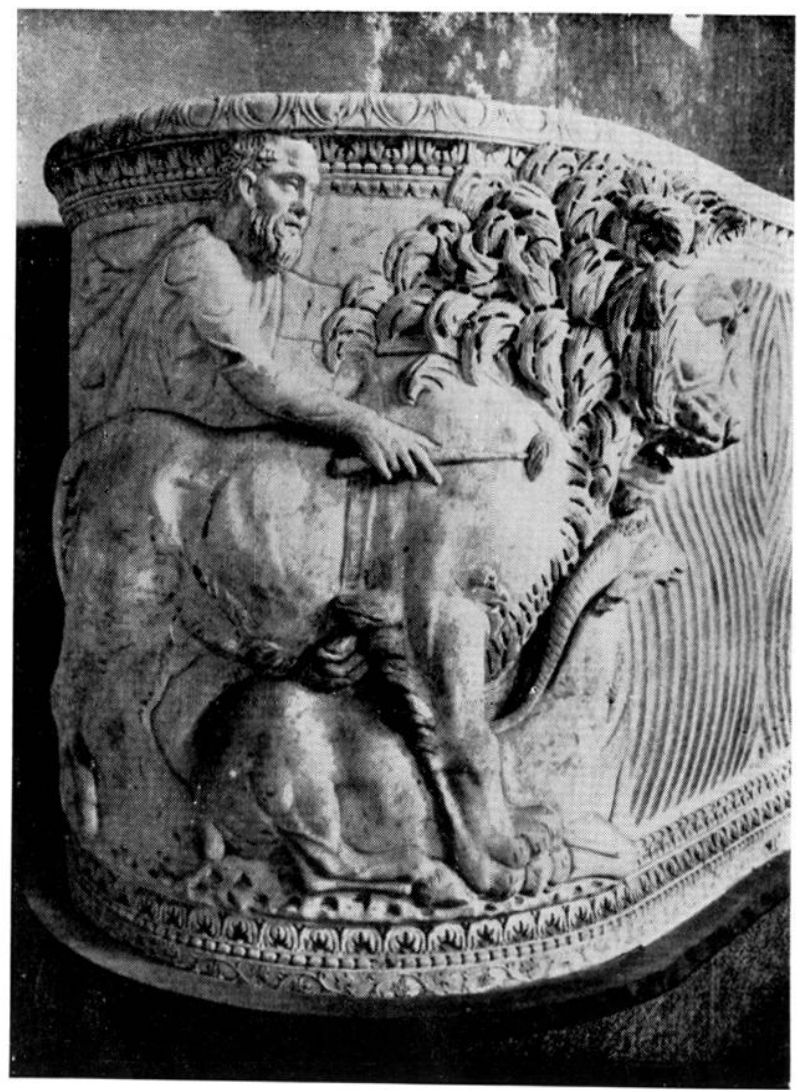

Fig. 1. - Sarcophaqe de la villa Tomlomia, Rome.

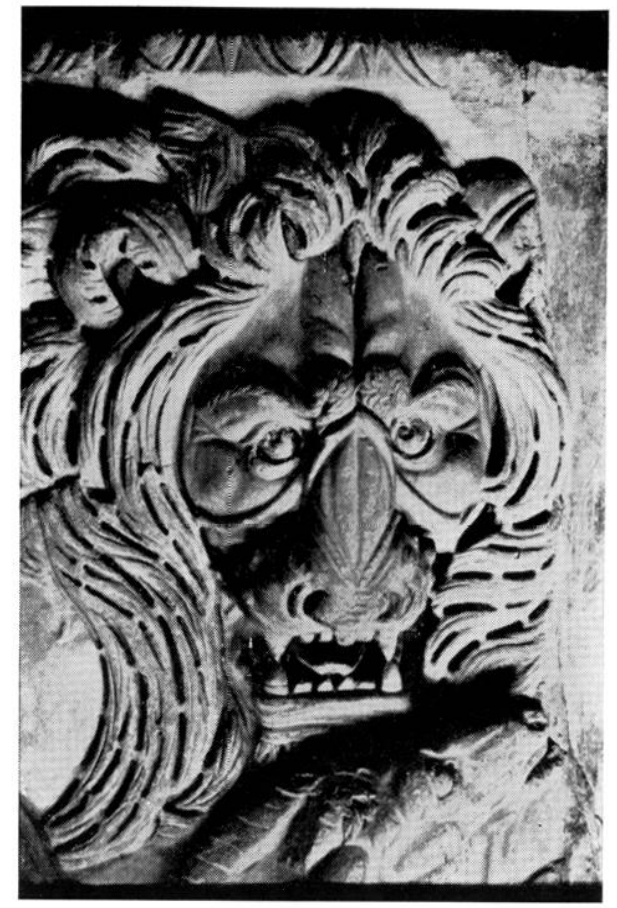

liig. D. Sarcophage de la villa Torlonia, Jome. rue sible avant nolre ire, lui a insulles une vie nouvelle (fig. 5). Gu on represenle parfois des animaux du cirque ne fait perut-ible qu'en préciser le sens. la ròle des compétilions de l'arine dans la vie el dans l'imagerie romaines éail immenseze ; lamphithécilre élail le poinl de rencontre. le centre des passions ef de l'interèl d'une grande partie des rilovens; a ses exhibitions sallachaienl des superstitions et des crovances profondément ancrées dans la

:? line itude ricente de .J. II. Sinomoxsox, The "Fancy Iress Banquel" dans Bullelin lan de lereeniging lol Bewerdering der Kemnis ran de antielie Beschaving le ." Grabenhage, XXX1. 1960, p. 25 a 55. a montri avec une remarquable precision ce robe des jeux du cirque. I. Auteur bludie, p. 52 fig. 2:3 et 21 , une terre cuite africaine ou le motif du lion terrassant un bovide figure dans un médaillon. Il est calque sur le revers dume monnaie de Tarse en Cilicie. du rione de (iordien III 238-9.14. Tont en itant lancien embleme de la cite asiatique. limage sur la terre cuite a sams ancum doute iti prise pour me secine de renalio de lon ancure. vie el dans la pensée conlemporaines. La vicloire au rirque etait considerée comme un présage de chance. comme un gage de bonheur pour celui qui avait misé sur la bìle gagnante. Le fauve viclorieux de notre mosaïque foomme ceux des sarcophages représente probablement re symbole de bon augure que le propriétaire de la maison tenail a montrer en bonne place a ses visileurs.

sur la lace antérieure de quatre sarcophagess de ce eroupe, les lions llanquent les quatre saisons. qui sont rassemblées aulour du défunt ${ }^{23}$. de mome que sur la mosaïque elles entourent le faure. Less svmboles du retour régulier des forres vives de la nalure se grou-

93: Voir Haxkmax, o. l., en particulier au t. II, p. 103. n. 14s. (e sont : un sarcophage du Palais des

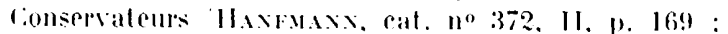
un autre d'Arricia, jardin de la villa chigri ibial. no $n^{\circ}$ 1. II. p. Ixt : un troisiome a la villa larpegna, Rome

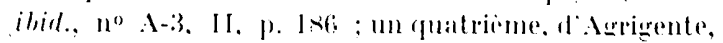
ibid., no.17+ h. 11, 1. 17\%. 
fent averes animanx, qui allirent la chance et proliegent rontre les forres du mal.

lóblude de ce parement mous apporle ainsi des precisions ehromologigues. ses élements ieomographiguess se lrourent rémis sur des monuments d'llalie de la premiere moitie da me sierele. (i. Rodenwald pernse que les premiers denler les sareophagess ans loms blaient plus anciens que les exemplaires conserves.

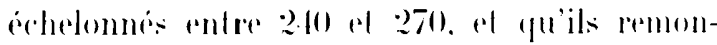

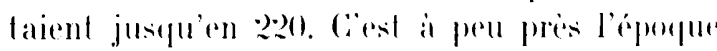
a lapuelle nous voudrions all ribuer la mosal̈gue.

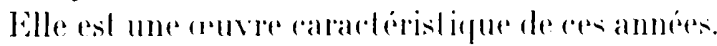

In autre lail encole se degage de nos obser-

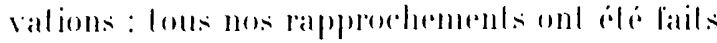

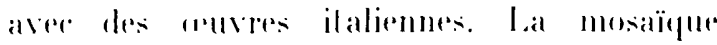
jurassienne as lres probablement la crealion d'un mailere venu direcelemenl de la péninsule. dinsi sexpliguent une composition el un proerammo icomographique exceptiommels dans la

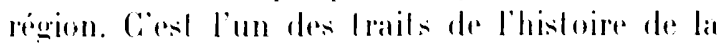
mosaïyue gallo-romaine. qure. dans une masse de produils banals. quelques-ums se signalent par des qualites pelu habituelles. La venue l'artistes ilaliens a dio donner de lempes a aul re une impulsion nowrelle a la produclion artisanale do pays.

Menri strix.

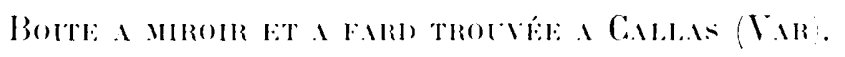

Iors de fomilles exerenteres all mois de sepe-

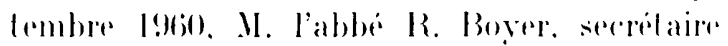

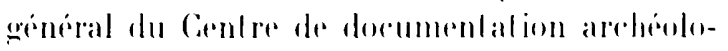
gique du Var, a ell ba bonne forlune de deconvrir un peelit objet forl interessant par lui-meme el par la calemorie. deja commue el cludiée. a layuelle il appartiente. Il sagil doune boile i

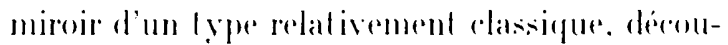
verte anx abords deme importante rilla walloromaine siluere an lien-dil "la Trinite". commume de Callas (Var. Lallenlion des fouilleurs avail ele alliren par les restes deun pelil momument quils onl pu dater de la fin

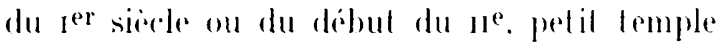
ou mausolée, transforme par la suile en chapelle des le $\mathrm{x}^{\mathrm{e}}$ sirele. Ciest an rours de fonilles

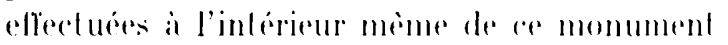
que la boile a miroir a ele derouverle. juste au-dessus d'un foyer an plare. (Yuelyues jours plus lard, M. l'abbe Boyer experdiail l'objel all

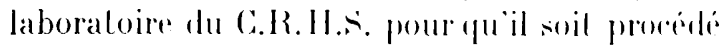
a son nellogage el si possible a son ouverture.

La boile se presentail sous la lorme d'un

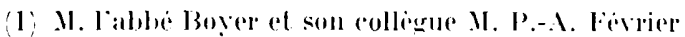
devant poursuive cette amme leurs foutless ont remis a une date ulterieure la publication de l'ensemble de leurs recherehes; quils trouvent ici l'expression de ma gratitude pour l'aimahle antorisation güils mont donnee, en me premettant de faire la presente publication. pelil disfue de bronze de fi centimel res environ

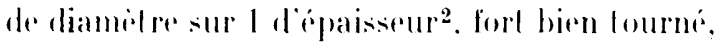
el erne en sen centere deune mennaie de bronze de Veron. sertie dans la fare superieure fles. 1 3) . lablal de conservalion élail des phos salis-

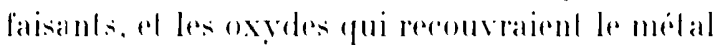
navaienl guere pénelre. si bien que la surface

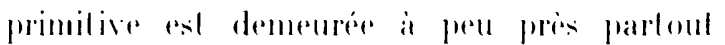

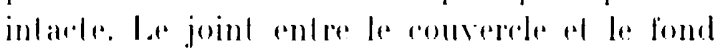

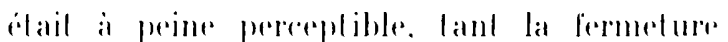

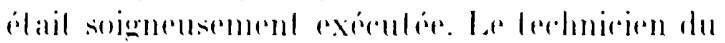
laboraboire. Il. Thouvenin. a pu insirer ume fine lame dans la fente. el par des pesies progressives a reussi asserz farilement el sans dommage a retirer le couverele. Quelle na pas ale alors nolere surprise en royant apparaitre. a lemvers du rouverele. In miroir admimablemenl conserere a peine lemi par une tres legeres

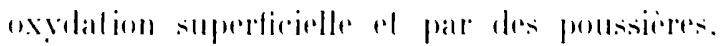

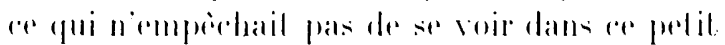

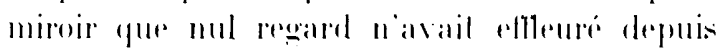
19) sireles. Ia fond de la boile contenail un minne depoil solide yui se dedachail parlielle-

(2) Ios mesures sont les suivantes : diamiolme maximum du comserele, ist mm, 5. hauteur du comserele

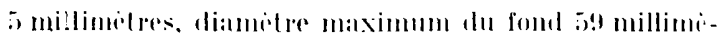

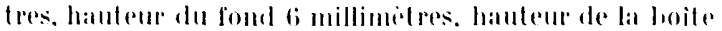

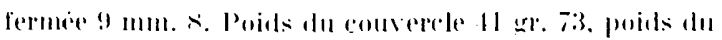
fond :3! 파. 73. 